\title{
Sustainable Business \\ International Journal
}

JULHO DE 2013- ISSN 1807-5908

COMPORTAMENTO DO CONSUMIDOR E SUSTENTABILIDADE: UMA

PERSPECTIVA BIBLIOMÉTRICA A LUZ DA BASE WEB OF SCIENCE E PERIÓDICOS CAPES

Valéria da Veiga Dias - DSc

Universidade Federal de Santa Maria

valeria-adm@ hotmail.com

Marcelo da Silva Schuster

Mestrando em Gestão de Organizações Pública

Universidade Federal de Santa Maria

marcelo.schuster@gmail.com

Luciana Flores Battistella -DSc

Universidade Federal de Santa Maria lutti@ufsm.br

Jean Philippe Palma Revillion- DSc

Universidade Federal do Rio Grande do Sul

jphir@ufrgs.br

\section{Resumo}

O objetivo do artigo apresentado foi verificar por meio de um levantamento com uso de pesquisa bibliométrica e teórica a condição das publicações referentes ao comportamento do consumidor e a sustentabilidade nos últimos 14 anos $(1998$ - 2012). O estudo foi viabilizado a partir da pesquisa na base de dados Web of Science e no portal Periódicos Capes. Analisou-se de forma quantitativa 291 artigos e qualitativamente os estudos mais relevantes disponíveis. Em relação a temática destaca-se a emergência das pesquisas sobre o tema salientando seu crescimento a partir de 2005 e a partir de 2010 quando foram identificados 142 artigos, o que reflete a realidade de transformações em termos de valores da sociedade e necessidades do cliente. Destaca-se a contribuição deste estudo já que apresenta um panorama de uma realidade mundial e que vem se consolidado no Brasil em termos de interesse do consumidor 


\title{
| Sustainable Business
}

JULHO DE 2013- ISSN 1807-5908

por modelos de gestão que envolvam propostas mais adequadas a promoção do equilíbrio com o meio ambiente no entanto tal proposta deve ser testada empiricamente por meio de modelos que trabalhem o comportamento do consumidor em relação aos valores e atitudes sustentáveis.

Palavras-chave: Comportamento do Consumidor, Sustentabilidade e Marketing

\begin{abstract}
The aim of this paper was presented to verify through a survey using bibliometric research and theoretical status of publications relating to consumer behavior and sustainability in the last 14 years (1998-2012). The study was made possible from the search in the database Web of Science, and the portal Portal Periodicos Capes. We analyzed 291 studies quantitatively and qualitatively the most relevant studies available. Regarding the theme highlights the emergence of research on the topic emphasizing its growth from 2005 and from 2010 when 142 articles were identified, reflecting the reality of transformations in terms of society's values and customer needs. Highlights the contribution of this study since it provides an overview of a global reality and that has been established in Brazil in terms of consumer interest in management models most suitable proposals involving the promotion of balance with the environment however this proposal should be tested empirically by means of models working consumer behavior towards sustainable values and attitudes.
\end{abstract}

Key Words: Consumer Behavior, Marketing and Sustainability 


\section{Sustainable Business International Journal}

\section{INTRODUÇÃO}

De acordo com MARIN; RUIZ (2007) embora a literatura voltada para o cliente e as relações com a empresa venham crescendo (Wulf et al, 2001), o ponto de vista do consumidor e a forma como se dão essas relações permanece inexplorada (Barnes,1997).

A melhoria na relação da empresa com o cliente e a promoção de um relacionamento de longo prazo com certeza apresenta resultados evidentes para a empresa em termos de rentabilidade(Stephens et al., 1996). Em compensação, para o consumidor, as motivações para a promoção desse relacionamento, bem como a manutenção do mesmo não apresentam resultados tão aparentes.

Recentemente a literatura revelou que um dos componentes motivadores para o envolvimento dos clientes em relacionamentos com empresas é ajudar as empresas a fornecer aos consumidores mais produtos e/ou serviços que satisfaçam as necessidades de indentificação com a mesma (AHEARNE et al, 2005;. BHATTACHARYA e SEN, 2003 apud MARIN E RUIZ, 2007).

Os pesquisadores Maignan e Ferrell (2004); Sen e Bhattacharya (2001) sugeriram que a teoria de identificação organizacional pode fornecer uma base sólida para a compreensão de como a identificação positiva promovida com o consumidor por meio do uso da responsabilidade social empresarial (RSE) gera o apoio ativo dos consumidores. Lichtenstein et al.(2004) seguindo esta linha destaca que o uso de modelo de Gestão Responsável ou que segue preceitos socioambientais gera maior possibilidade de identificação positiva do consumidor e prolongamento do relacionamento. 


\section{Sustainable Business International Journal}

JULHO DE 2013- ISSN 1807-5908

Considerando tais possibilidades e teorias que visam explicar o envolvimento do consumidor a partir de questões sustentáveis, sociais ou socioambientais é que se dá o foco deste estudo que consiste em um levantamento e análise bibliométrico visando apresentar um panorama das publicações da relação entre o comportamento do consumidor e sustentabilidade com uso da base de dados Web of Science e Periódicos Capes. Optou-se ainda por uma análise qualitativa dos estudos mais relevantes, considerando a quantidade de citações existentes.

\section{MARCO TEÓRICO E ESTUDOS ANTERIORES}

O estudo do consumidor parte de uma premissa básica: cada indivíduo é "um mundo à parte”, ou seja, tem características e experiências únicas. A primeira decisão para uma estratégia de marketing é a definição do público-alvo da empresa através da estratégia de segmentação, que é a identificação e a seleção de grupos de consumidores relativamente homogêneos. Isto se torna necessário, pois os mercados são heterogêneo, compostos por pessoas diferentes, com desejos e expectativas distintas (LIMEIRA, 2007).

Considerando esta perspectiva cabe ressaltar que toda a atividade econômica é, em última análise destinada a satisfazer as necessidades dos consumidores, sendo assim, o comportamento do consumidor tem um papel essencial para a promoção de qualquer transição para uma economia mais sustentável. Para se aproximar de uma sociedade sustentável, os consumidores terão de reduzir o seu nível de consumo e / ou modificar os tipos de bens que consomem, o que se associa a proposta do uso de bens tecnológicos, em alguns casos de forma positiva e em outros de forma negativa. No entanto, não está claro como a redução do nível global de consumo individual podem ser induzidos. Considerando os aparentemente 


\section{Sustainable Business International Journal}

JULHO DE 2013- ISSN 1807-5908

insaciáveis desejos humanos, uma redução voluntária é improvável de acontecer, especialmente como o aumento da produtividade e da inovação tecnológica que continuamente abre novas oportunidades de consumo. (BUENSTORF E CORDES, 2008).

Recentemente a literatura revelou que um dos componentes motivadores para o envolvimento dos clientes em relacionamentos com empresas é ajudar as empresas a fornecer aos consumidores mais produtos e/ou serviços que satisfaçam as necessidades de indentificação com a mesma (AHEARNE et al, 2005;. BHATTACHARYA e SEN, 2003 apud MARIN E RUIZ, 2007).

Os pesquisadores Maignan e Ferrell (2004); Sen e Bhattacharya (2001) sugeriram que a teoria de identificação organizacional pode fornecer uma base sólida para a compreensão de como a identificação positiva promovida com o consumidor por meio do uso da responsabilidade social empresarial (RSE) ou outros modelos responsáveis gera o apoio ativo dos consumidores. Lichtenstein et al.(2004) seguindo esta linha destaca que o uso de modelo de Gestão Responsável ou que segue preceitos socioambientais gera maior possibilidade de identificação positiva do consumidor e prolongamento do relacionamento.

A proposta de inserção e modificação da cultura do consumo emergiu a partir das décadas de 1960 e 1970 quando algumas nações passaram a observar os efeitos nocivos da poluição e de uma série de acidentes que causaram diversos tipos de impactos ambientais (qualquer alteração físico química ou biológica causada pelo homem ao ambiente, pessoas, comunidades, biota). Em decorrência disto foram levantadas diversas discussões (Clube de Roma- 1960), foram realizados eventos como a Conferência do Estocolmo (1972), elaborados tratados e protocolos visando alertar sobre as necessidades de transformações nas relações de consumo e no relacionamento do homem com seu ambiente. 


\section{Sustainable Business International Journal}

JULHO DE 2013- ISSN 1807-5908

Segundo Dias(2011) a década de 80 constituiu-se como um momento de implantação de legislação e estudos técnicos em diversas nações, mas apenas em 1987 com a publicação do "Nosso Futuro Comum- relatório de Relatório Brundtland da Comissão Mundial sobre Meio Ambiente e Desenvolvimento (CMAD) formalizou-se o que à muito tempo era apenas uma tentativa de elaborar metas e apresentar propostas sobre sustentabilidade. O relatório apresenta o conceito de desenvolvimento sustentável como: "desenvolvimento que satisfaz as necessidades do presente sem comprometer a capacidade das gerações futuras para satisfazer suas próprias necessidades" (WCED, 1987, p. 42).

A análise do relatório demonstrou claramente a insustentabilidade de padrões existentes de desenvolvimento, produção e consumo( Nascimento, 2008). Enquanto isso, a preocupação sobre as questões ambientais e sociais entre os consumidores cresceu, bem como as expectativas de que esta preocupação influenciam comportamento do consumidor (Vandermerwe e Oliff, 1990; Worcester, 1993 apud Peattie e Peattie (2009), e esse comportamento deveria ser um estímulo para a inovação e o desenvolvimento de novos produtos. No entanto, é notável que apenas substituir produtos e campanhas não é suficiente para a incorporação de transformações da sociedade e na sociedade de consumo, portanto cabe destacar o papel do Marketing como uma possibilidade na inserção destas novas práticas socio ambientais para a promoção de uma cultura de sustentabilidade.

Os primeiros estudos relacionados ao consumo ambiental e social surgiram ainda na década de 1970 e abordaram prioritariamente as questões ambientais e seus impactos na sociedade. Alguns estudiosos inseriram a idéia de ecologia aos estudos do Marketing(Henion e Kinnear,1976) com a proposta até mesmo de uma teoria do Consumo Consciente apresentada por Fisk(1973). No entanto tais estudos perderam força em virtude de proposta de 


\section{Sustainable Business International Journal}

crescimento industrial e crises envolvendo potências nacionais da época e só foram retormadas após a divulgação do Relatório de Brundtland em 1987.

O crescimento dos debates sobre tais questões trouxeram foram geradoras de um incentivo aos produtos verdes e o entendimento da participação da sociedade no boicote aos produtos com CFC e seus derivados (Elkington e Hailes, 1988) o que trouxe uma enxurada de tentativas de Marketing Verde, Ambiental e Sustentável (Fuller, 1999) na tentativa de inserção de produtos diferenciados associados as estratégias corporativas.

No entanto, de acordo com Peattie e Peattie (2009) tais mudanças comportamentais representaram mais consumo responsável, mas a ênfase na substituição de compra em favor de produtos ambientais nunca foi uma possibilidade de fazer uma contribuição substancial para a sustentabilidade. A forma de comercialização que está crescendo rapidamente, e tem considerável potencial para contribuir para a redução do consumo, é o Marketing Social (que é diferente de, mas infelizmente frequentemente confundido com, marketing societal). $\mathrm{O}$ marketing social trabalha a partir da mudança cultural, de comportamento baseado em objetivos sociais mas a partir do entendimento do marketing tradicional. O termo foi cunhado por Kotler e Zaltman (1971) como o uso de princípios e técnicas de marketing para promover uma idéia ou comportamento social, ou seja, atua junto a proposta do comportamento do consumidor e do relacionamento da organização com o mesmo.

Marin e Ruiz (2006) também estudaram a temática do comportamento do consumidor considerando a capacidade que a organização tem de atrair o consumidor em virtude de sua identidade organizacional, já enfatizando novos valores presentes no perfil do consumidor atual. Evidenciou-se no estudo destes autores, com uso de instrumento validado, que a contribuição do modelo de Responsabilidade Social Empresarial foi maior do que a 


\section{Sustainable Business International Journal}

Capacidade Empresarial, ou seja, foi mais atrativa ao consumidor considerando sua identificação com a identidade da empresa. O conceito de identificação, segundo Mael e Ashforth, 1992, foi desenvolvido nas áreas de psicologia social e comportamento organizacional, e satisfaz a necessidade de identidade social e auto-definição, sendo influenciador também da lealdade do consumidor e colaborador.

Identificou-se que os consumidores, assim como respondem à premissa social, favorecem a sustentabilidade nas duas dimensões estudadas( econômica e ambiental) e preços, dando avaliações positivas e a intenção de compra para empresas. Além disso, em comparação, os consumidores respondem mais negativamente a empresa que não é sustentável do que o respondem mais positivamente às empresas com maior preocupação com a sustentabilidade econômica. Os consumidores não respondem favoravelmente aos baixos preços, quando eles têm informações sobre o baixo envolvimento da empresa com a sustentabilidade ambiental. Finalmente, os consumidores avaliam uma empresa mais favorável se ela possui ações de causas sociais. Em geral os consumidores respondem a múltiplas dimensões da sustentabilidade de forma positiva(CHOI, NG, 2011).

Essa condição do ambiente promove a criação estruturas de comunicação, melhoria dos modelos de segmentação de mercado e estratégias de marketing de relacionamento e marketing direto. Esta tendência para a relação de comercialização está ocorrendo em parte porque a tecnologia da informação dá à empresa a capacidade de personalizar seus serviços e desenvolver relações com clientes (RUST, 2004). Portanto, o estudo das caracteristicas do ambiente a partir da perspectiva do consumidor visa o entendimento destes processos de mudança, pressões e influência para adoção de comportamentos organizacionais específicos, 


\section{Sustainable Business International Journal}

sendo eles relacionados ao perfil do consumidor e ou das organizações presentes no campo organizacional.

\section{METODOLOGIA}

A bibliometria, técnica escolhida para este estudo permite situar a pesquisa através de diversos indicadores e diversas relações. Como indicadores podem ser usados o número de trabalhos, número de citações, co-autoria, número de patentes, número de citações de patentes, bem como podem ser feitos mapas dos campos científicos e dos países (MACIASCHAPULA, 1998, p. 137 apud OLIVEIRA e BRANCO, 2012).

De acordo com Araújo (2006), a área mais importante da bibliometria é a análise de citações, a qual contribui para o desenvolvimento da ciência, além de prover o necessário reconhecimento de um cientista outros cientistas, estabelecer os direitos de propriedade e prioridade da contribuição científica de um autor, constituir importante fonte de informação, ajudar a julgar os hábitos de uso da informação e mostrar a literatura que é indispensável para o trabalho científico (FORESTI, 1989).

\subsection{Coleta de dados}

Para a construção do referencial teórico deste estudo sobre o comportamento do consumidor foi realizada uma pesquisa na base Web of Science com o topic "consumer behavior" para os últimos 14 anos (1998 a 2012) e foram encontrados 2.746 estudos. Dentre as categorias da base de pesquisa e as áreas de pesquisa, a temática ambiental aparece entre as 10 primeiras na qual o estudo do comportamento do consumidor mais publica. 


\section{Sustainable Business International Journal}

JULHO DE 2013- ISSN 1807-5908

A partir deste primeiro levantamento realizada no dia 17 de maio de 2013 e optou-se ainda por um refinamento considerando os hot topics "consumer behavior" and "sustainability”. Foram encontrados então 41 estudos relacionando os dois temas. Estes estudos foram classificados conforme "Times Cited - highest to low west" ou seja, os estudos mais citados, visando encontrar os trabalhos com maior relevância diante do tema. Realizouse a mesma busca no portal Periódicos Capes, ou seja, buscou-se os termos "consumer behavior" e "sustainability", no entanto nesta ferramenta não foi possível buscar os termos de forma associada, o que resultou em 1370 estudos. Ressalta-se que essa pesquisa utilizou a proxy de uma universidade federal visando maior acesso aos documentos encontrados. Realizou-se posteriormente um refinamento por temas: Sustainable Behavior, Consumer Preferences, Consumer Research, Sustainability, Sustainable Development e Consumer Behavior.

Refinou-se ainda as publicações por tipo de documento, selecionando apenas artigos, o que resultou em 291 artigos. Primeiramente verificou-se se entre estes estudos estavam os 39 já encontrados e constatou-se que todos os estudos encontrados na primeira pesquisa ( Web of Science) foram identificados na segunda pesquisa ( periódicos). Analisou-se de forma quantitativa todos os 291 artigos e qualitativamente apenas os artigos disponíveis considerando os 10 primeiros listados em ordem de relevância relacionando os dois temas de alguma forma. De acordo com esse refinamento foram realizadas as considerações sobre os estudos mais citados para cada temática e inferidas as análises sobre o consumidor visando caracterizar a participação dos fatores sustentáveis.

\subsection{Análise de dados}




\section{Sustainable Business International Journal}

JULHO DE 2013- ISSN 1807-5908

A análise dos dados sustentou-se nas abordagens de pesquisa qualitativa e quantitativa. Em termos qualitativos, foram analisados os assuntos tratados nas 10 publicações pesquisadas consideradas mais relevantes, de acordo com a ordem de citações( mais citado ao menos citado). Quanto aos dados quantitativos, procurou-se investigar as seguintes variáveis: total de publicações, os autores, áreas temáticas, tipos de documentos, título das fontes, ano das publicações, idiomas, países e análise do número de vezes que cada publicação foi citada através do índice h-b e do índice m.

Hirsch em 2005 apresenta então o h-index (ou índice h) que mede o impacto por meio da quantidade de citações, o fator h-index é baseada na profundidade de anos da sua subscrição do produto e seu período de tempo selecionado( livre tradução Web of Science, 2013). Banks (2006) contribuiu com o índice h-b, uma extensão do $h$-index e é obtido por meio do número de citações de um tópico ou combinação em determinado período, listados em ordem decrescente de citações. Ele é encontrado em publicações que tenham obtido um número de citações igual ou maior à sua posição no ranking. Banks (2006) também explica o cálculo do índice $\mathrm{m}$, o qual é obtido mediante a divisão do índice $\mathrm{h}-\mathrm{b}$ pelo período de anos que se deseja obter informações (n).

Para a análise dos índices h-b e m, foram utilizadas as considerações de Banks (2006):

\begin{tabular}{|l|l|}
\hline Indicador & \multicolumn{1}{c|}{ Descrição } \\
\hline $0<\mathrm{m} \leq 0,5$ & $\begin{array}{l}\text { o tópico/combinação pode ser de interesse para pesquisadores em um campo específico de } \\
\text { pesquisa, onde o campo engloba uma comunidade pequena }\end{array}$ \\
\hline $0,5<\mathrm{m} \leq 2$ & $\begin{array}{l}\text { o tópico/combinação provavelmente pode ser um "hot topic" como área de pesquisa, onde a } \\
\text { comunidade é muito grande ou o tópico/combinação apresenta características muito } \\
\text { interessantes }\end{array}$ \\
\hline $\mathrm{m} \geq 2$ & $\begin{array}{l}\text { é um tópico/combinação exclusivo, onde as consequências têm um alcance não apenas na } \\
\text { sua própria área de pesquisa. É provável que seja um tópico/combinação com efeitos de } \\
\text { aplicação ou características únicas. }\end{array}$ \\
\hline
\end{tabular}




\section{Sustainable Business \\ International Journal}

JULHO DE 2013- ISSN 1807-5908

Considerando as definições de Banks (2006) neste estudo serão considerados hot topics as combinações com índice $\mathrm{m} \geq 2$. A partir destes foram elaborados os resultados teóricos do estudo em questão.

\section{RESULTADOS}

Considerando os estudos pesquisados foram condensados os principais dados e contribuições em termos teóricos. Os autores que mais publicaram sobre a interligação dos assunto consumidor e sustentabilidades foram, Verbeke, Vanhonacker, I Ali, Ha-Brookshire JE, Jackson T, Uzzell D, Banerjee e Solomon, Buenstorf e Cordes, Peattie e Peattie cada um com duas publicações.

Faz-se importante referenciar que a relação sobre o tema tem chamado atenção dos pesquisadores, uma vez que as publicações apresentaram um crescimento maior a partir de 2005 conforme Figura 1 que apresenta os últimos 14 anos de publicações (consideram-se publicações até 2012, pois 2013 é o ano corrente):

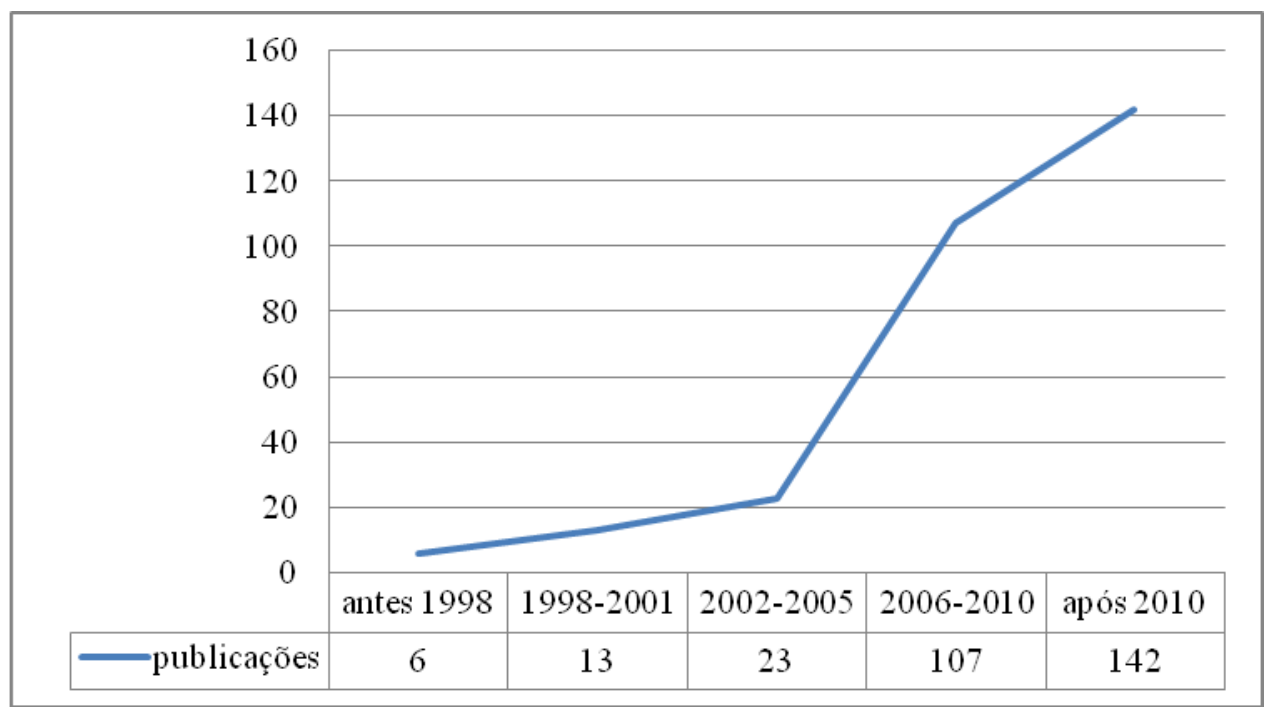




\section{Sustainable Business \\ International Journal}

JULHO DE 2013- ISSN 1807-5908

Figura: Evolução das publicações sobre comportamento do consumidor e sustentabilidade nos últimos 14 anos.

Do total de documento analisados 100\% deles são artigos (291). Em relação aos países que concentram as publicações sobre o assunto é possível observar que os Estados Unidos da América se destaca como país com maior número de publicações na área concentrando para a temática, seguido da Inglaterra e Holanda e representam juntos $70 \%$ das publicações analisadas. Destas publicações evidenciou-se também que 94\% do material publicado concentrou-se na língua inglesa, $1 \%$ em espanhol e o restante em Chinês, Português e Francês.

Sobre o consumidor e a sustentabilidade a exploração do tema ficou por conta de Clothing And Textiles Research Journal, Ecological Economics, Journal of Business Ethics cada um com 6 publicações e Amfiteatru Economic, Fashion Theory The Journal Of Dress Body Culture, European Journal of Marketing e Journal Of Industrial Ecology com 4 publicações cada. Considerando as áreas de Publicação 44\% do total concentra-se na área de Economia e Negócios e 33\% Ecologia Ambiental conforme figura 2 a seguir: 


\section{Sustainable Business International Journal}

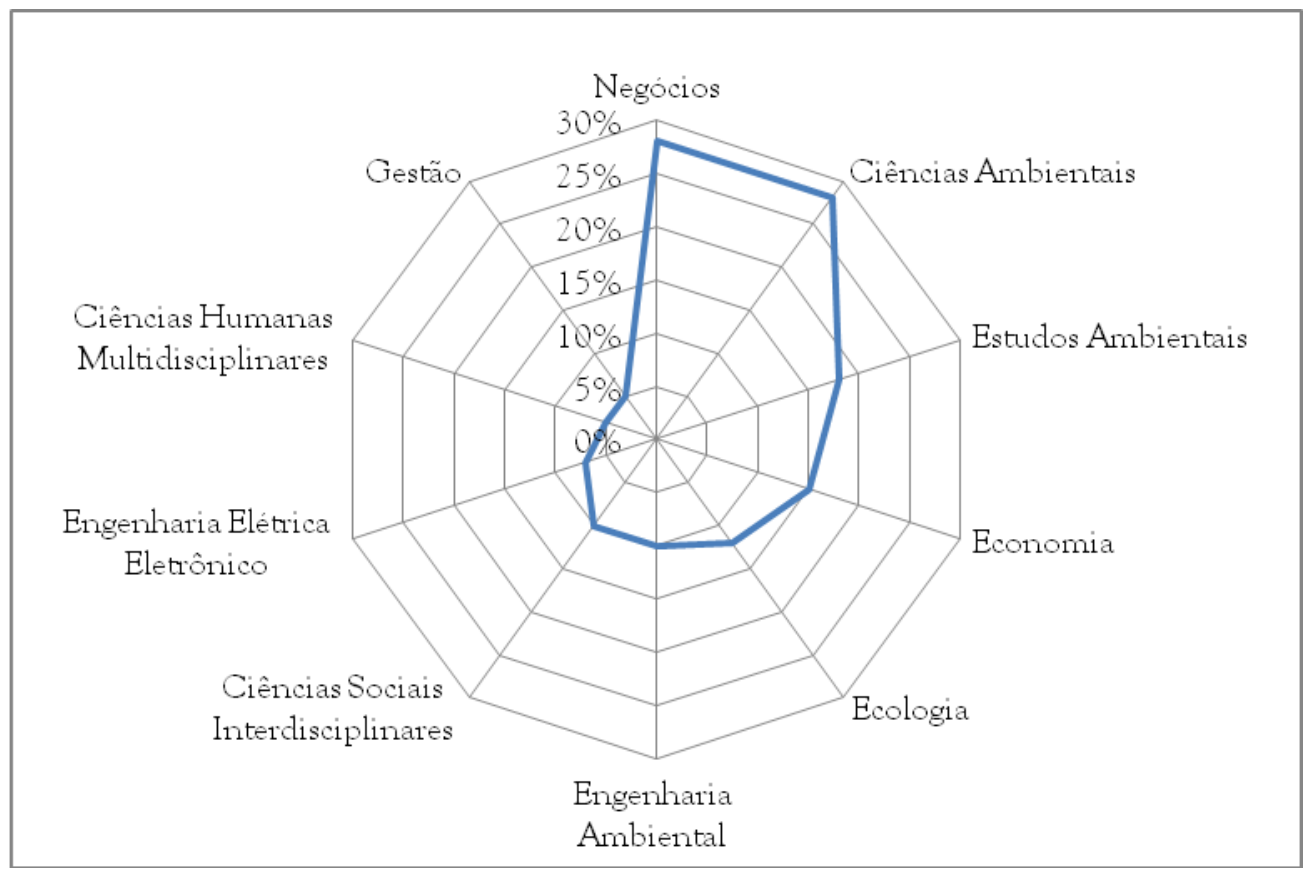

Figura 2 - Áreas de publicação

Para os artigos analisados identificou-se um total de 190 citações no período estudado sendo que average citations item ou a média de citações foi de 4,63 e $h$-index 8 . Contribuiriam para o $h$-index apenas os 8 primeiros artigos listados. Visando a classificação em termos de relevância os artigos selecionados por quantidade de citações tiveram seu índice b e coeficiente $m$ calculados. Estes também foram analisados de forma qualitativa, sendo que três destes artigos não estavam disponíveis para consulta livre, portanto outros artigos também listados na sequiência foram utilizados para compor AA análise qualitativa.

\begin{tabular}{|l|l|c|c|}
\hline \multicolumn{1}{|c|}{ Título } & \multicolumn{1}{|c|}{ Autor } & Índice-b & coeficiente-m \\
\hline $\begin{array}{l}\text { Social marketing: A pathway to } \\
\text { consumption reduction? }\end{array}$ & $\begin{array}{l}\text { Peattie, Ken; Peattie, } \\
\text { Sue }\end{array}$ & 36 & 2,6 \\
\hline $\begin{array}{l}\text { Eco-labeling for energy efficiency } \\
\text { and sustainability: a meta-evaluation } \\
\text { of US programs A; }\end{array}$ & $\begin{array}{l}\text { Banerjee, } \\
\text { Solomon, BD }\end{array}$ & 34 & 2,4 \\
\hline $\begin{array}{l}\text { The Impacts of Household } \\
\text { Consumption and Options for }\end{array}$ & $\begin{array}{l}\text { Tukker, Arnold; } \\
\text { Cohen, Maurie J.; }\end{array}$ & 15 & 1,1 \\
\hline
\end{tabular}




\section{Sustainable Business International Journal}

JULHO DE 2013- ISSN 1807-5908

\begin{tabular}{|c|c|c|c|}
\hline Change & $\begin{array}{l}\text { Hubacek, Klaus; et } \\
\text { al. }\end{array}$ & & \\
\hline 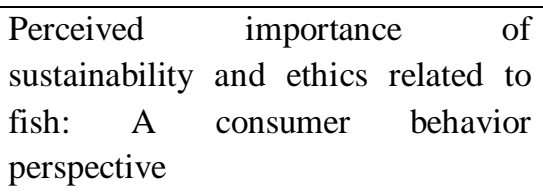 & $\begin{array}{l}\text { Verbeke, Wim; } \\
\text { Vanhonacker, Filiep; } \\
\text { Sioen, Isabelle; et al. }\end{array}$ & 15 & 1,1 \\
\hline $\begin{array}{lll}\text { Eco-Friendly Attitudes: } & \text { What } \\
\text { European Citizens Say and What } \\
\text { They Do }\end{array}$ & $\begin{array}{l}\text { Pirani, E.; Secondi, } \\
\text { L. }\end{array}$ & 11 & 0,8 \\
\hline $\begin{array}{l}\text { Can sustainable consumption be } \\
\text { learned? A model of cultural } \\
\text { evolution }\end{array}$ & $\begin{array}{l}\text { Buenstorf, Guido; } \\
\text { Cordes, Christian }\end{array}$ & 10 & 0,7 \\
\hline $\begin{array}{l}\text { Consumers' } \\
\text { associations favoring free-range meat } \\
\text { or less meat }\end{array}$ & $\begin{array}{l}\text { de Boer, Joop; } \\
\text { Boersema, Jan } \quad \text { J.; } \\
\text { Aiking, Harry }\end{array}$ & 9 & 0,6 \\
\hline $\begin{array}{l}\text { Strengths and limitations of } \\
\text { localizing food production as a } \\
\text { sustainability-building strategy - an } \\
\text { analysis of bread production on the } \\
\text { island of Gotland, Sweden }\end{array}$ & $\begin{array}{lr}\text { Sundkvist, } & \mathrm{A} ; \\
\text { Jansson, } & \mathrm{AM} ; \\
\text { Larsson, } \mathrm{P} & \end{array}$ & 9 & 0,6 \\
\hline
\end{tabular}

Comprova-se a relevância dos estudos ao verificar que todos têm o coeficiente $\mathrm{m}$ acima de 0,5 e os dois primeiros artigos podem ser considerados hot topics.

\section{Contribuição qualitativa}

Em relação as contribuições teóricas segue o que foi destacado: Peattie e Peattie (2009) apresentam reflexões sobre as direções do consumo se forem motivadas por idéias de sustentabilidade e promovidas com uso do Marketing Social. Os autores consideram o potencial do marketing para contribuir para a redução do consumo a partir de uma perspectiva de marketing social. Os autores analisam as dificuldades de aplicação da teoria do marketing convencional em busca de um consumo mais sustentável, e a lógica da aplicação de uma forma adaptada como o marketing social visando promover estilos de vida mais sustentáveis e reduções no consumo. A mesma idéia da associação com práticas de cidadania e 


\section{Sustainable Business International Journal}

responsabilidade social Sheth; Sethia e Srinivas (2011) identificam reações de consumo positivas ao associar empresas e consumo.

Banerjee e Solomon(2003) vislumbraram seu estudo a partir da perspectiva do consumidor e seu entendimento da abordagem do uso dos rótulos ecológicos, que resulta em melhoria do desempenho ambiental por meio da escolha do consumidor. Enquanto a rotulagem ecológica em si não é novo, eco-rotulagem visa promover a eficiência ou sustentabilidade e é um fenômeno mais recente. O estudo comprovou que um simples selo de aprovação de logotipos e etiquetas em geral afetam mais o comportamento do consumidor do que os complexos rótulos de divulgação de informações. Tal resultado abre a discussão sobre a importância do Marketing verde e do destaque ao estudo e desenvolvimento das propostas organizacionais por meio da marca.

Buenstorf e Cordes (2008) também estudam um aprofundamento da idéia do Marketing Verde a partir da reflexão sobre a aprendizagem de padrões de consumo sustentável. Consideram que estes padrões podem se espalhar dentro de uma população via processos de aprendizagem social, embora um viés de aprendizagem individual forte pode favorecer produtos nocivos para o ambiente. Os autores apresentam um modelo que mostra como a transmissão transmissão tendenciosa de comportamentos diferentes por meio de influencia o comportamento de consumo.

Koh e Lee (2012) afirmam que embora mais os consumidores estejam exigindo produtos mais "verde" e associados a produção sustentável, o consumismo verde e melhoria das práticas de produção, por si só pode não adequadamente reduzir a destruição das florestas e da biodiversidade. Afirmam ainda que as mudanças no comportamento do consumidor nos 


\section{Sustainable Business International Journal}

JULHO DE 2013- ISSN 1807-5908

países industrializados poderiam substancialmente aliviar os impactos ambientais associados à produção agrícola nos trópicos em desenvolvimento.

Doorn e Verhoef (2011) afirmam que em 2008, o alimento orgânico gerava uma receita de quase 51.000 milhões dólares (Biomonitor, 2009). O mercado de alimentos orgânicos cresce em aumento e a atenção do consumidor para as questões ambientais e de bem-estar animal, consciência devido à extensa cobertura na mídia e crescentes de consequiências da poluição ambiental, o aquecimento global, e o uso de recursos naturais. Outro motivo para a escolha do consumidor produtos orgânicos diz respeito a questões de saúde. Alarmados por dramaticamente crescentes taxas de obesidade e discussões sobre segurança alimentar, muitas consumidores desejam mais saudável, menos processados, alimentos naturais (MARKETWATCH, 2008). Alguns autores citam a preservação da saúde e melhora, em vez de motivos éticos, como as principais razões os consumidores compram produtos orgânicos (MCEACHERN e MCCLEAN, 2002; SCHIFFERSTEIN e OUDE OPHUIS, 1998).

Cherrier; Black; Lee (2011) estudaram tendências ao anticonsumo ou não consumi baseado na proposta de consumir apenas produtos saudáveis ou de organizações com comportamento sustentável. O estudo identificou que as práticas de não consumo e retaliação formam um quadro complementar entre aqueles que valorizam esse tipo de produto e ação empresarial.

Mariadoss; Tansuhaj; Mouri (2011) apontam descobertas e desenvolvimento de um arcabouço conceitual que liga as capacidades de marketing para estratégias de inovação e sustentabilidade com o comportamento de consumo sustentável e empresa vantagem competitiva, e indicam proposições para futuras pesquisas. Apontam questões relacionadas a 


\section{Sustainable Business International Journal}

JULHO DE 2013- ISSN 1807-5908

idéia de que para influenciar consumidores a adotar produtos ou marcas que são ambientalmente amigáveis, as empresas precisam de capacidades de marketing especializados que podem efetivamente relacionar esta informação ao entendimento do consumidor, tornando o produto atrativo. As empresas precisam trabalhar com uso de idéias inovativas, isso porque os consumidores estão cada vez mais céticos em relação as mensagens verdes.

\section{CONSIDERAÇÕES FINAIS}

Em virtude dos dados encontrados e do referencial teórico construído pode-se inferir sobre a relevância da temática estudada em termos de inserção na proposta prática do entendimento do comportamento do consumidor. Nota-se uma grande quantidade de estudos empíricos e teóricos abordando de diversas formas os temas sustentabilidade e comportamentos do consumidor.

A relevância do tema se comprova pelo crescimento do interesse de pesquisa e pelas transformações inseridas no contexto de produtos e serviços do mercado atual. De acordo com os estudos analisados verificou-se também que há uma diversidade de propostas e conceitos que permeiam esta discussão como o marketing verde, o consumo de produtos orgânicos, etc.

Destaca-se a contribuição deste estudo já que apresenta um panorama de uma realidade mundial e que vem se consolidado também no Brasil em termos de interesse do consumidor por modelos de gestão que envolvam propostas mais adequadas a promoção do equilíbrio com o meio ambiente no entanto tal proposta deve ser testada empiricamente por meio de modelos que trabalhem o comportamento do consumidor em relação aos valores e atitudes sustentáveis. Nota-se ainda uma quantidade de estudos na área de negócios envolvendo alimentos, alimentos orgânicos, estratégias de marketing e reflexões sobre a 


\section{Sustainable Business \\ International Journal}

JULHO DE 2013- ISSN 1807-5908

incorporação do tema de forma mais estável dentro da agricultura, indústrias de transformação e organizações.

Como sugestão para estudos futuros fica o indicativo de focar a pesquisa em estudos brasileiros e temáticas isoladas, já que este estudo que é um levantamento, aponta diversas vertentes que podem produzir resultados relacionados tanto de forma empírica como teórica.

\section{REFERÊNCIAS}

ARAÚJO, C. Bibliometria: evolução histórica e questões atuais. Em Questão, 12, 2006.

BANERJEE, Abhijit, SOLOMON, Barry. Eco-labeling for energy efficiency and sustainability: a meta-evaluation of US programs. Energy Policy n.31, 109-123, 2003.

BANKS, M. 2006. An Extension of the Hirsch index: indexing scientific topics and compounds. Disponível em: 〈http://www.arxiv.org/abs/physics/0604216> Acesso em: 13/02/2013.

BUENSTORF, Guido, CORDES, Christian. Can sustainable consumption be learned? A model of cultural evolution. Ecological Economics n.6 7, 2008.

CHERRIER Helene, BLACK, Iain R., LEE, Mike. Intentional non-consumption for sustainability: Consumer resistance and/or anti-consumption? European Journal of Marketing, v. 45, p.1757 - 1767, 2011.

CHOI, S. NG. A. Environmental and Economic Dimensions of Sustainability and Price Effects on Consumer Responses. Journal of Business Ethics, n.104 v.2 p.269 -282, 2011. DOI 10.1007/s10551-011-0908-8 


\section{Sustainable Business International Journal}

DOORN, Jenny van e VERHOEF, Peter C. Willingness to pay for organic products: Differences between virtue and vice foods. Intern. J. of Research in Marketing, n. 28. p.167-180, 2011.

ELKINGTON J, HAILES J. The green consumer guide. London: Victor-Gollanz, 1988.

FISK G. Criteria for a theory of responsible consumption. JMark, n.37, v.2, p.24-31, 1973.

FULLER D. Marketing mix design-for-environment: a systems approach. J Bus Adm Policy Anal, p.309-39, 1999.

GATERSLEBEN, B., STEG L., VLEK C. Measurement And Determinants Of Environmentally Significant Consumer Behavior, Environment And Behavior, v. 34 n. 3, 2002.

HENION KE, KINNEAR TC. Ecological marketing, In: American Marketing Association's First National Workshop on Ecological Marketing, 1976.

$\mathrm{KOH}$ Lian Pin, LEE Tien Ming. Sensible consumerism for environmental sustainability. Biological Conservation, p.151, 2012).

KOTLER P, ZALTMAN G. Social marketing: an approach to planned social change. J Mark, n. 35, p.3-12, 1971.

MARIADOSS Babu John, TANSUHAJ Patriya Silpakit, MOURI Nacef Marketing capabilities and innovationbased strategies for environmental sustainability: An exploratory investigation of B2B firms. Industrial Marketing Management. v. 40, p.1305-1318, 2011. MARIN, Longinos, RUIZ, Salvador. 'I Need You Too!' Corporate Identity Attractiveness for Consumers and The Role of Social Responsibility. Journal of Business Ethics, n.71, p.245- 260, 2007. 


\section{Sustainable Business International Journal}

NASCIMENTO, L.; LEMOS, Â; MELLO. M. Gestão Sócio-Ambiental Estratégica. Porto Alegre: Bookman, 2008.

OLIVEIRA Natália G de, BRANCO Zuleika. Estudo Bibliométrico da Produção Científica da Universidade Federal de Minas Gerais: publicações no Web of Science no período de 2004 a 2008. Disponível em: < http://pt.scribd.com/doc/37961490/Analise-BibliometricaUFMG> Acesso em: 21/07/2012.

PEATTIE Ken, PEATTIE, Sue. Social marketing: A pathway to consumption reduction? Journal of Business Research, n.62 p.260-268, 2009.

PEHNT Martin. Dynamic life cycle assessment (LCA) of renewable energy Technologies. Renewable Energy n.31, 2006.

SHETH Jagdish N; SETHIA Nirmal K.; SRINIVAS Shanthi. Mindful consumption: a customer-centric approach to sustainability. Journal of the Academy of Marketing Science, n.39 v.1 p.21, 2011. DOI 10.1007/s11747-010-0216-3

STEPHENS, D. L., R. P. HILL and K. Bergman Enhancing The Consumer-Product Relationship: Lessons From the QVC Home Shopping Channel. Journal of Business Research, n.37, v.3, p.193-202, 2006. 